\title{
Efecto de la concentración de poliacrilamida sobre la calidad del depósito de cobre obtenido por refino electrolítico( $\left.{ }^{\circ}\right)$
}

\author{
J. Aragón*, J. Ipinza**, J. Camus***, A. Vargas*y R. Saavedra*
}

Resumen

\begin{abstract}
El efecto del aditivo poliacrilamida no iónica (PAM), se estudió a nivel de laboratorio, como afinador de grano cristalino en el refino electrolítico del cobre. Se realizaron experimentos de electrólisis de $24 \mathrm{~h}$, utilizando un electrolito de sulfato de cobre ácido, con 0,5 a $10 \mathrm{mgl}^{-1}$ de poliacrilamida, a $55^{\circ} \mathrm{C}$ y una densidad de corriente de $260 \mathrm{Am}^{-2}$. La efectividad de este aditivo orgánico en la refinación electrolítica del cobre fue determinada mediante la medida de la rugosidad de la superficie del depósito de cobre y contrastada con los resultados de otras técnicas instrumentales (MEB y análisis metalográfico). Los resultados obtenidos del análisis MEB, análisis metalográfico y medición de la rugosidad superficial del depósito de cobre, muestran que a concentraciones tan bajas como $0,5 \mathrm{mgl}^{-1}$ de este aditivo, se manifiesta su propiedad de afinador de grano. Sin embargo, en el rango de 7 a $10 \mathrm{mgl}^{-1}$ de poliacrilamida, se alcanza un tamaño de grano óptimo para el depósito de cobre en el cátodo.
\end{abstract}

\section{Effect of the polyacrylamide concentration on the quality of the copper deposit obtained by electrolytic refining}

\begin{abstract}
The effect of the additive non ionic polyacrylamide (PAM), was studied at the laboratory level, as refining crystalline grain in the copper electrorefining. Experiments were performed $24 \mathrm{~h}$ of electrolysis, using an electrolyte of copper sulphate acid, with 0.5 to $10 \mathrm{mgl}^{-1}$ polyacrylamide at $55^{\circ} \mathrm{C}$ and a current density of $260 \mathrm{Am}^{-2}$. The effectiveness of these organic additives in copper electrorefining was determined by directly measuring the surface roughness of the copper deposit and contrasted with the results of other instrumental techniques (SEM and metallographic analysis). The results of the analysis SEM, metallographic analysis and measurement of the surface roughness of the copper deposit, show that to concentrations as low as $0.5 \mathrm{mgl}^{-1}$ of this additive is manifested its property of tuner of grain. However, in the range of 7 to $10 \mathrm{mgl}^{-1}$ of polyacrylamide, it reaches an optimum size of grain for the deposit of copper in the cathodes.
\end{abstract}

Keywords Copper cathodes; Polyacrylamide; Grain size; Grain refining.

\section{INTRODUCCIÓN}

La electrodeposición, etapa final de la cadena de valor de obtención de cátodos de cobre, es una operación que presenta una gran complejidad debido al gran número de fenómenos involucrados y variables operacionales que intervienen en el proceso. En particular, mantener la composición del electrolito en condiciones óptimas, que permitan la obtención de un depósito de alta calidad, conlleva grandes dificultades, que no sólo están relacionadas con la concentración de ion cúprico, de ácido e impurezas, sino además, con la elección del tipo de aditivo afinador de grano y determinación de su rango de concentración más adecuado. Un verdadero problema, a nivel industrial es mantener constante la concentración del agente afinador en la disolución electrolítica ${ }^{[1]}$.

Por largo tiempo, en el proceso de refino electrolítico de cobre se han utilizado una combinación de aditivos, principalmente tiourea, gelatina, cola y cloruro $^{[2}$ y 3$]$. En electro-obtención, en cambio, mayoritariamente la industria ha utilizado la goma "guar", polisacárido de la familia de los galactamananos que se comercializa bajo diversos nombres comerciales,

(•) Trabajo recibido el día 22 de abril de 2013 y aceptado en su forma final el día 21 de agosto de 2013.

* Facultad de Ingeniería y Arquitectura, Universidad Arturo Prat, Av. Arturo Prat 2120, Iquique, Chile, E-mail: juan.aragon@unap.cl

** Facultad de Ingeniería, Universidad Técnica Federico Santa María, Av. España 1680, Valparaíso, Chile, E-mail: Jorge.ipinza@usm.cl

*** Facultad de Ciencias, Universidad de Playa Ancha, Av. Gran Bretaña 40, Playa Ancha, Valparaíso, Chile, E-mail: jcamus@upla.cl 
con el cual se logra una cristalización de cobre de grano fino, compacto y orientado en la dirección del campo eléctrico, a concentraciones del orden de $4 \mathrm{mgl}^{-1}{ }^{[4-6]}$. No obstante, se han investigado otros compuestos, que siendo prometedores, no se han aplicado industrialmente ${ }^{[7-9]}$. En la búsqueda de aditivos afinadores de menor costo que la goma "guar", se ha probado la modificación de polisacáridos ${ }^{[10]}$. Recientemente, ha empezado a aplicarse en algunas plantas de electroobtención de Chile, un aditivo inhibidor, registrado bajo el nombre de DXG-F7, correspondiente a una dextrina, que promete resultados aun superiores a los alcanzados con la goma "guar" [11].

Vereecken y Winand ${ }^{[12]}$, investigaron el compuesto poliacrilamida a fines de la década del setenta. Las principales características descritas para este compuesto en disolución de ácido sulfúrico son: constituir moléculas de elevado peso molecular, 500.000 a 12.000.000 u.m.a., adoptar la estructura catiónica en este medio y a concentraciones tan bajas como $1 \mathrm{mgl}^{-1}$ reducir el tamaño de grano cristalino. Estudios posteriores realizados por Fabian et al. ${ }^{[13}$ y 14] , indican que el compuesto poliacrilamida, activado en disolución ácida diluida (en una dieciseisava parte) y agregado al electrolito en el rango de concentración desde 0,01 hasta $10 \mathrm{mgl}^{-1}$, genera depósitos de grano más fino que los obtenidos con "guar".

\section{PARTE EXPERIMENTAL}

\subsection{Determinación del rango óptimo de concentración de poliacrilamida}

Los experimentos se realizaron a nivel de laboratorio, en un conjunto de tres celdas construidas en material acrílico (3), de doble camisa para mantener la temperatura de trabajo $\left(55^{\circ} \mathrm{C}\right)$ con agua caliente (4), cada una de un volumen de $300 \mathrm{ml}$. Se utilizaron cátodos de acero inoxidable $316 \mathrm{~L}$, de dimensión $5 \times 5 \mathrm{~cm}$, y ánodos de cobre electrolítico de igual área. En cada celda se dispuso un cátodo y un ánodo ubicados en forma vertical y paralelamente entre ellos. La alimentación eléctrica se realizó con una fuente de poder de marca HP, modelo 92048 (1). La circulación del agua caliente se logró usando un termocirculador de marca Julabo (4). La instalación experimental se muestra en la figura 1.

Los experimentos realizados durante $24 \mathrm{~h}$, tuvieron como finalidad determinar el rango de concentración del aditivo PAM, necesario para obtener un depósito de cobre de calidad óptima, dejando constantes la densidad de corriente, la composición del electrolito base y la temperatura.

La calidad de los cátodos de cobre obtenidos se evaluó midiendo la rugosidad del depósito, contras-

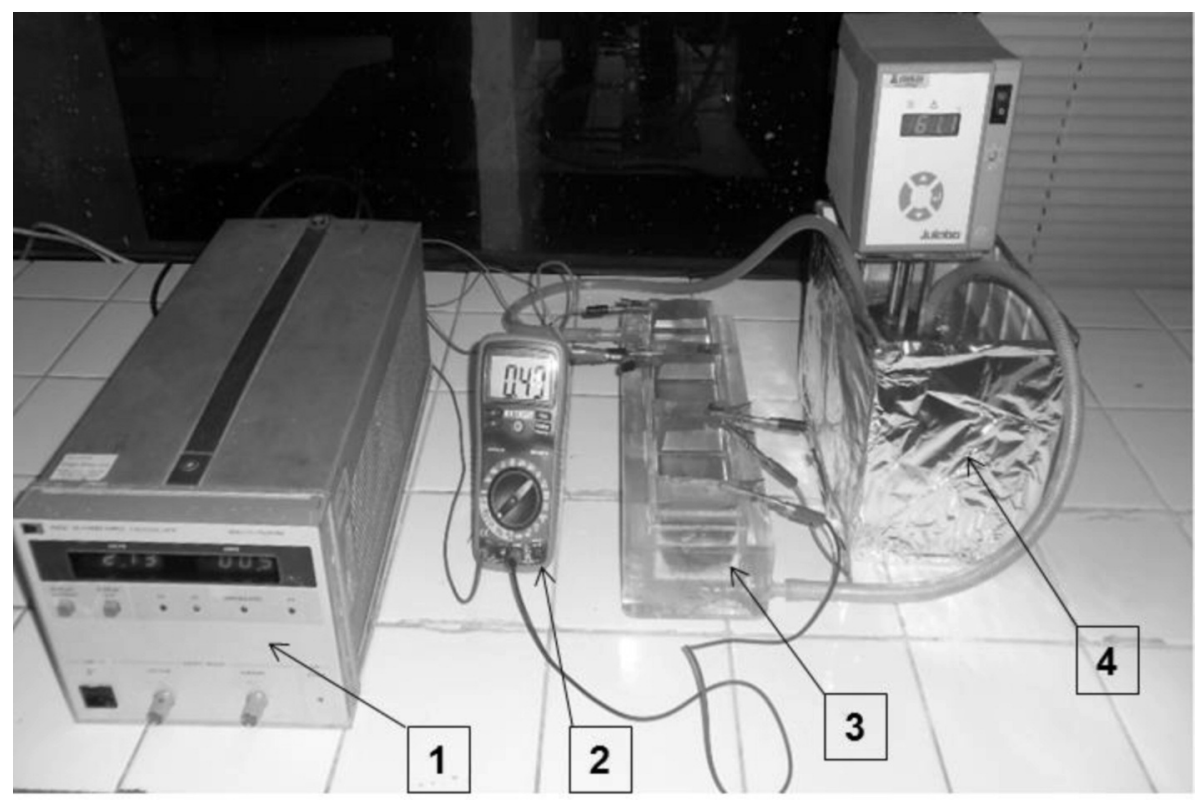

Figura 1. Instalación experimental de los equipos para el desarrollo de los experimentos: (1) fuente de poder; (2) voltímetro digital; (3) celdas construidas en material acrílico; (4) baño termocirculador de agua.

Figure 1. Installation of equipment in the laboratory for the development of the experiments: (1) power supply; (2) digital voltmeter; (3) cells manufactured in acrylic material; (4) bath water thermocirculator. 
tando los resultados con los obtenidos mediante análisis metalográfico y MEB (ampliación de X200). En la tabla I se describe la composición del electrolito preparado, utilizando reactivos grado para análisis Merck, para cada una de las electrólisis programadas. Se utilizó en este estudio poliacrilamida no iónica donada por la empresa CYTEC Chile.

Se pesó la cantidad requerida de aditivo PAM, seguidamente se disolvió en electrolito base y se acondicionó agitando mecánicamente durante $1 \mathrm{~h}$, adicionándolo a la celda para realizar la electrólisis de 24 h. Durante la electrólisis, a manera de control, cada dos horas se registró el potencial catódico y el voltaje de celda mediante un voltímetro digital (ver (2) en Fig. 1). Al término de cada experimento, se retiraron los cátodos, se lavaron con agua destilada, alcohol y se secaron con aire caliente.

Los depósitos catódicos obtenidos en los experimentos fueron analizados por MEB, análisis metalográfico y mediciones de la rugosidad superficial.

Cortes representativos de cátodos de $0,5 \times 1 \mathrm{~cm}^{2}$ aproximadamente fueron analizados mediante MEB. Para el análisis metalográfico, se muestrearon cortes de $2 \times 0,5 \mathrm{~cm}^{2}$ en las láminas de cobre obtenidas experimentalmente, que se fijaron sobre briquetas de resina transóptica.

Se realizaron mediciones de rugosidad de las láminas catódicas utilizando un rugosímetro, SRT-6200. Los puntos de medición se determinaron mediante el diseño de una plantilla, como una forma de estandarizar la medida. En cada punto de la superficie, se realizaron 30 mediciones de $\mathrm{Rz}$ y $\mathrm{Ra}$, utilizando para el muestreo una longitud de 2,5 mm con 5 puntos de muestreo.

Ra es el valor de rugosidad media aritmética medido en micrones, reconocido y utilizado internacionalmente. Es el valor medio aritmético de los valores absolutos de las variaciones del perfil dentro del tramo de medición. El valor numérico medido es siempre menor al valor $\mathrm{Rz}$ obtenido en el mismo perfil de rugosidad. $\mathrm{Rz}$ es la profundidad de la rugosidad media medida en micrones, que corresponde a la media aritmética de las mayores profundidades de rugosidad por separado de diferentes tramos de medición colindantes.

\subsection{Determinación del efecto de la poliacrilamida sobre la polarización de la semi-reacción de reducción del ion cúprico}

Se utilizó una celda electroquímica de vidrio, con electrodo auxiliar de platino, un electrodo de referencia de $\mathrm{Hg} / \mathrm{Hg}_{2} \mathrm{SO}_{4} / \mathrm{K}_{2} \mathrm{SO}_{4}$ saturado y un electrodo de disco rotatorio de cobre de $5 \mathrm{~mm}$ de diámetro, marca Radiometer Analitical modelo EDI101. La velocidad de rotación se mantuvo en $1.200 \mathrm{rpm}$, regulada mediante una unidad de control de velocidad marca Radiometer Analitical Modelo CTV101. Se aplicó una velocidad de barrido de $5 \mathrm{mVs}^{-1}$ utilizando un potenciostato Voltalab, modelo PGP201. Las curvas de polarización se trazaron utilizando el elec-

Tabla I. Composición y acondicionamiento del electrolito de trabajo

Table I. Composition and conditioning of the work electrolyte

\begin{tabular}{|c|c|c|}
\hline Parámetro & Unidad & Valor \\
\hline Concentración de $\mathrm{Cu}^{2+}$ & $\mathrm{gl}^{-1}$ & 45 \\
\hline Concentración de $\mathrm{H}_{2} \mathrm{SO}_{4}$ & $\mathrm{gl}^{-1}$ & 180 \\
\hline $\begin{array}{l}\text { Concentración poliacrilamida } \\
\text { (Variable según prueba) }\end{array}$ & $\mathrm{mgl}^{-1}$ & $0,5-10$ \\
\hline $\begin{array}{l}\text { Temperatura de acondicionamiento } \\
\text { de PAM en electrolito }\end{array}$ & ${ }^{\circ} \mathrm{C}$ & 55 \\
\hline $\begin{array}{l}\text { Tiempo de acondicionamiento de } \\
\text { PAM en electrolito }\end{array}$ & $\mathrm{h}$ & 1 \\
\hline Preparación de aditivo PAM & - & Disuelto en electrolito base \\
\hline $\begin{array}{l}\text { Preparación de aditivo "guar" } \\
\text { Galactasol } \AA\end{array}$ & - & $\begin{array}{l}\text { Disuelto en agua tibia } \\
\text { durante } 45 \text { min, luego, } \\
\text { agregando al electrolito } \\
\text { base alcanzando la } \\
\text { concentración de } 4 \mathrm{mgl}^{-1}\end{array}$ \\
\hline
\end{tabular}


trolito base, con distintas concentraciones de aditivo (guar o poliacrilamida).

Previo al trazado de cada curva, durante $15 \mathrm{~min}$, el electrolito fue sometido a un burbujeo con gas nitrógeno de alta pureza.

\section{RESULTADOS Y DISCUSIÓN}

\subsection{Efecto de la concentración del aditivo poliacrilamida}

La figura 2 (a), muestra una imagen MEB en la que aparece el depósito catódico de cobre obtenido sin aditivo. Se observa una superficie poco uniforme, con nódulos de gran magnitud, en que el diámetro del grano cristalino supera frecuentemente los $200 \mu \mathrm{m}$. En la figura 2 (b), se observa que la adición de $4 \mathrm{mgl}^{-1}$ de galactasol, produce en comparación con la condición sin aditivo (Fig. 2 (a)), una disminución significativa del tamaño de grano cristalino, obteniéndose un depósito fino, compacto y con un nivel menor de nodulación.

Las figuras 3 (a) a 3 (h), muestran el estado superficial de los depósitos de cobre obtenidos cuando se agregan distintas concentraciones de PAM. Pequeñas dosis del aditivo del orden de $0,5 \mathrm{mgl}^{-1}$, producen una disminución del tamaño del grano cristalino. Sin embargo, en el intervalo de 3 a $10 \mathrm{mgl}^{-1}$ de PAM, el depósito de cobre muestra una significativa disminución de la nodulación, incrementando gradualmente la uniformidad de la estructura, fineza y compactación.

La figura 3 (f) muestra que con $8 \mathrm{mgl}^{-1}$ de PAM, se alcanzan características similares al depósito obtenido con $4 \mathrm{mgl}^{-1}$ de galactasol ${ }^{\circledR}$ (Fig. 2 (b)).
Los resultados obtenidos mediante el análisis metalográfico a partir de las mismas muestras catódicas, refuerzan los resultados anteriores.

La figura 4 (a), depósito obtenido sin aditivos, muestra una cristalización gruesa, dejando de manifiesto un depósito tipo BR, según la clasificación de Fischer ${ }^{[15]}$. La figura 4 (b), muestra el perfil de crecimiento del depósito de cobre obtenido con $3 \mathrm{mgl}^{-1}$ de PAM. El relieve superficial presenta mayor uniformidad y, aun cuando se observa una cristalización gruesa, su ancho es notoriamente menor, con una reducción en promedio desde 200 a $80 \mu \mathrm{m}$. Se observa además, una mayor orientación de los cristales en la dirección del campo eléctrico, coincidiendo con la aparición de cristales poliédricos sin orientación.

La figura 4 (c), muestra el depósito obtenido con $4 \mathrm{mgl}^{-1}$ de PAM. Se nota un efecto más marcado del aditivo, obteniéndose una estructura cristalina orientada y bastante más compacta, principalmente en la zona inferior y media del cátodo de cobre, pero al final del crecimiento, se aprecia un incremento en el grosor de los cristales, aun cuando éste se ha reducido en comparación con los depósitos presentados en las figuras 4 (a) y 4 (b). El depósito de cobre obtenido con $5 \mathrm{mgl}^{-1}$ de PAM, (Fig. 4 (d)), muestra un relieve superficial bastante uniforme; la cristalización muestra una estructura más heterogénea y menos compacta, sin embargo, el ancho promedio de los cristales continúa reduciéndose.

El depósito con $6 \mathrm{mgl}^{-1}$ de PAM, (Fig. 4 (e)), no presenta cambios significativos con respecto al obtenido con $5 \mathrm{mgl}^{-1}$ (Fig. 4 (d)), aunque en promedio, el ancho de los cristales continúa disminuyendo.

La placa de cobre obtenida con $7 \mathrm{mgl}^{-1}$ de PAM (Fig. 4 (f)), muestra una mayor afinación del grano, generándose una mejor orientación al campo eléctrico y además una superficie mucho más uniforme.
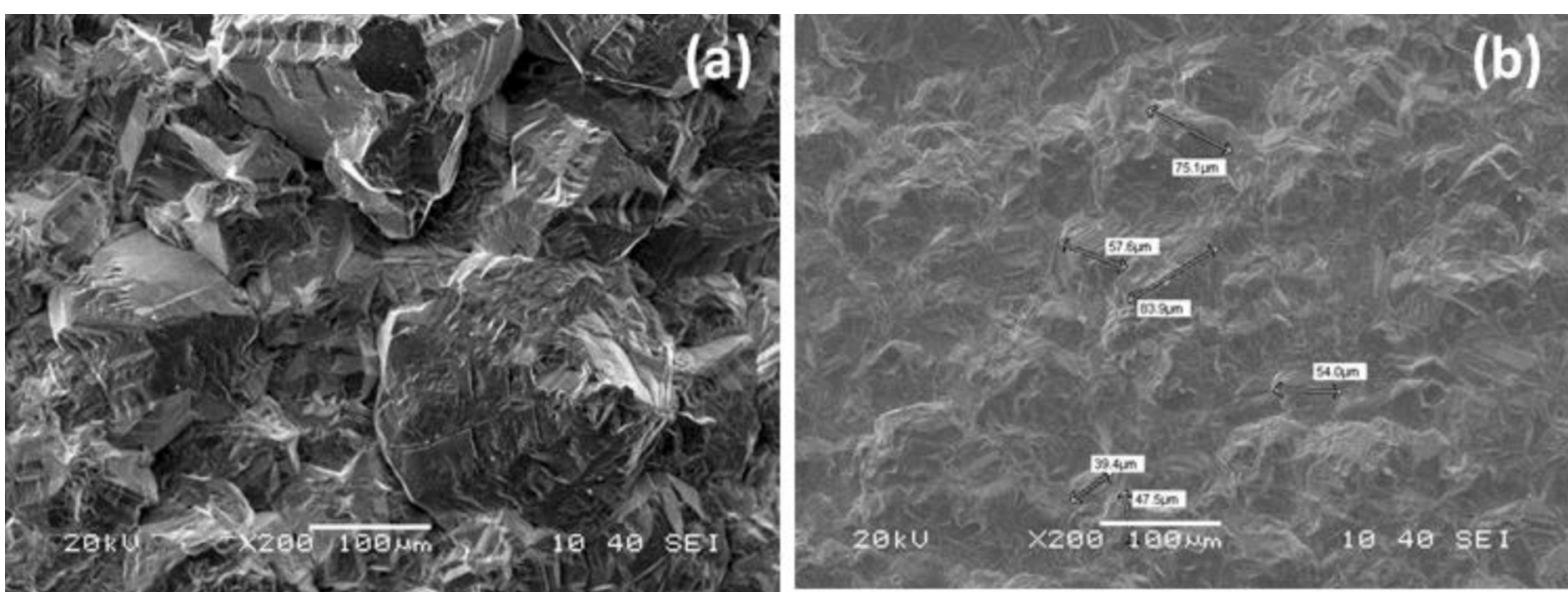

Figura 2. Superficie del depósito de cobre: (a) sin aditivo; (b) con $4 \mathrm{mgl}^{-1}$ de galactasol.

Figure 2. Surface of copper deposit: (a) without additives; (b) with $4 \mathrm{mgl}^{-1}$ of additive galactasol. 

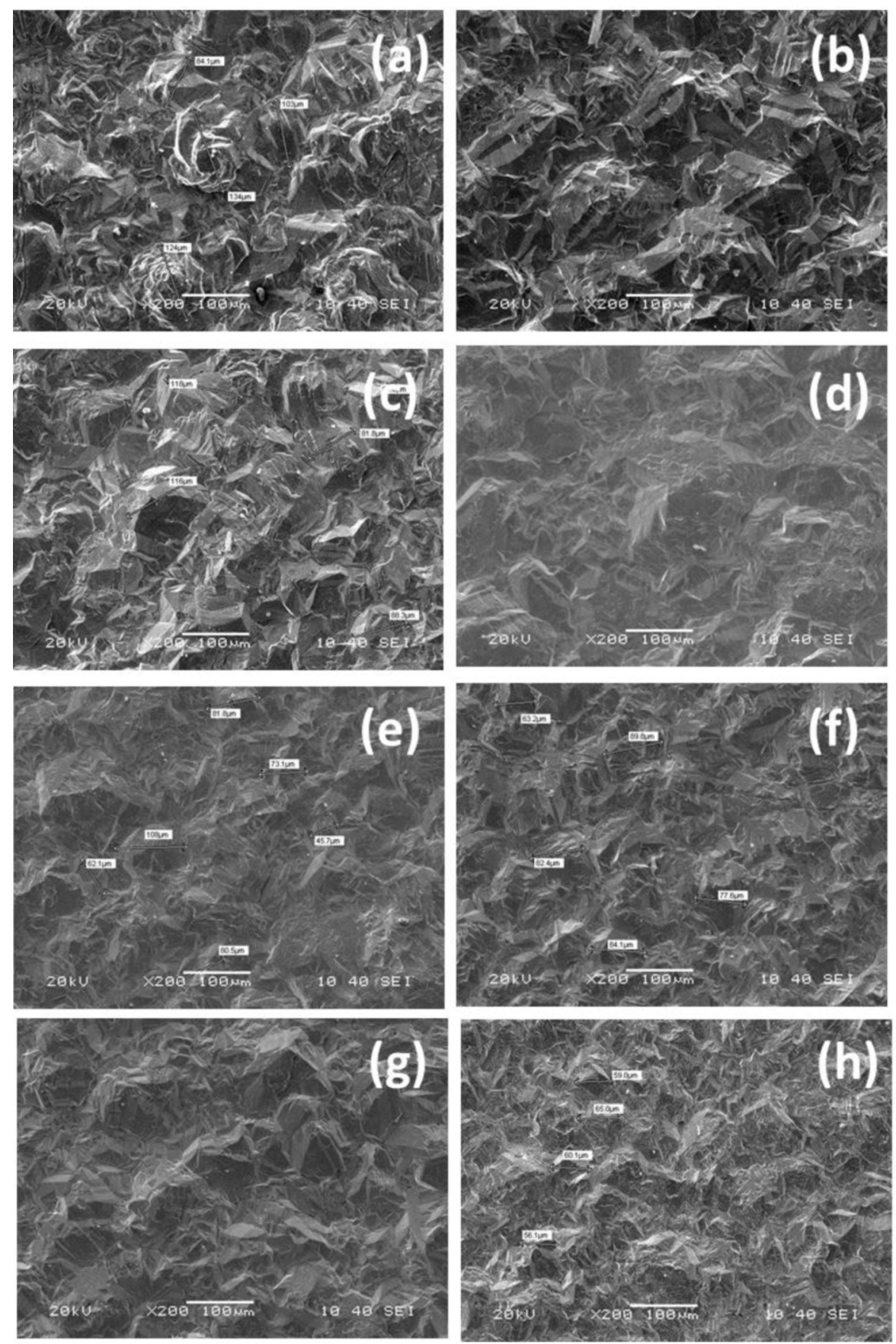

Figura 3. Superficie del depósito de cobre con diferentes concentraciones de PAM: (a) $3 \mathrm{mgl}^{-1}$; (b) $4 \mathrm{mgl}^{-1}$; (c) $5 \mathrm{mgl}^{-1}$; (d) $6 \mathrm{mgl}^{-1}$; (e) $7 \mathrm{mgl}^{-1}$; (f) $8 \mathrm{mgl}^{-1} ;$ (g) $9 \mathrm{mgl}^{-1} \mathrm{y}$ (h) $10 \mathrm{mgl}^{-1}$ (X200).

Figure 3. Surface of copper deposit with different concentration of PAM:

(a) $3 \mathrm{mgl}^{-1}$; (b) $4 \mathrm{mgl}^{-1}$; (c) $5 \mathrm{mgl}^{-1}$; (d) $6 \mathrm{mgl}^{-1}$; (e) $7 \mathrm{mgl}^{-1}$; (f) $8 \mathrm{mgl}^{-1}$;

(g) $9 \mathrm{mgl}^{-1}$ and (h) $10 \mathrm{mgl}^{-1}$ (X200).

El depósito con $8 \mathrm{mgl}^{-1}$ de PAM, mostrado en la figura $4(\mathrm{~g})$, exhibe una gran homogeneidad en su crecimiento orientado en el campo y una uniformidad óptima del relieve superficial, indicativos para clasificar el depósito como del tipo FT. El perfil del grano, muestra una estructura bastante compacta, no observándose irregularidades intercristalinas.

Los depósitos con $9 \mathrm{mgl}^{-1}$ (Fig. 4 (h)) y $10 \mathrm{mgl}^{-1}$ (Fig. 4 (i)) de PAM, respectivamente, presentan estructuras compactas y orientadas en el campo. Sin 

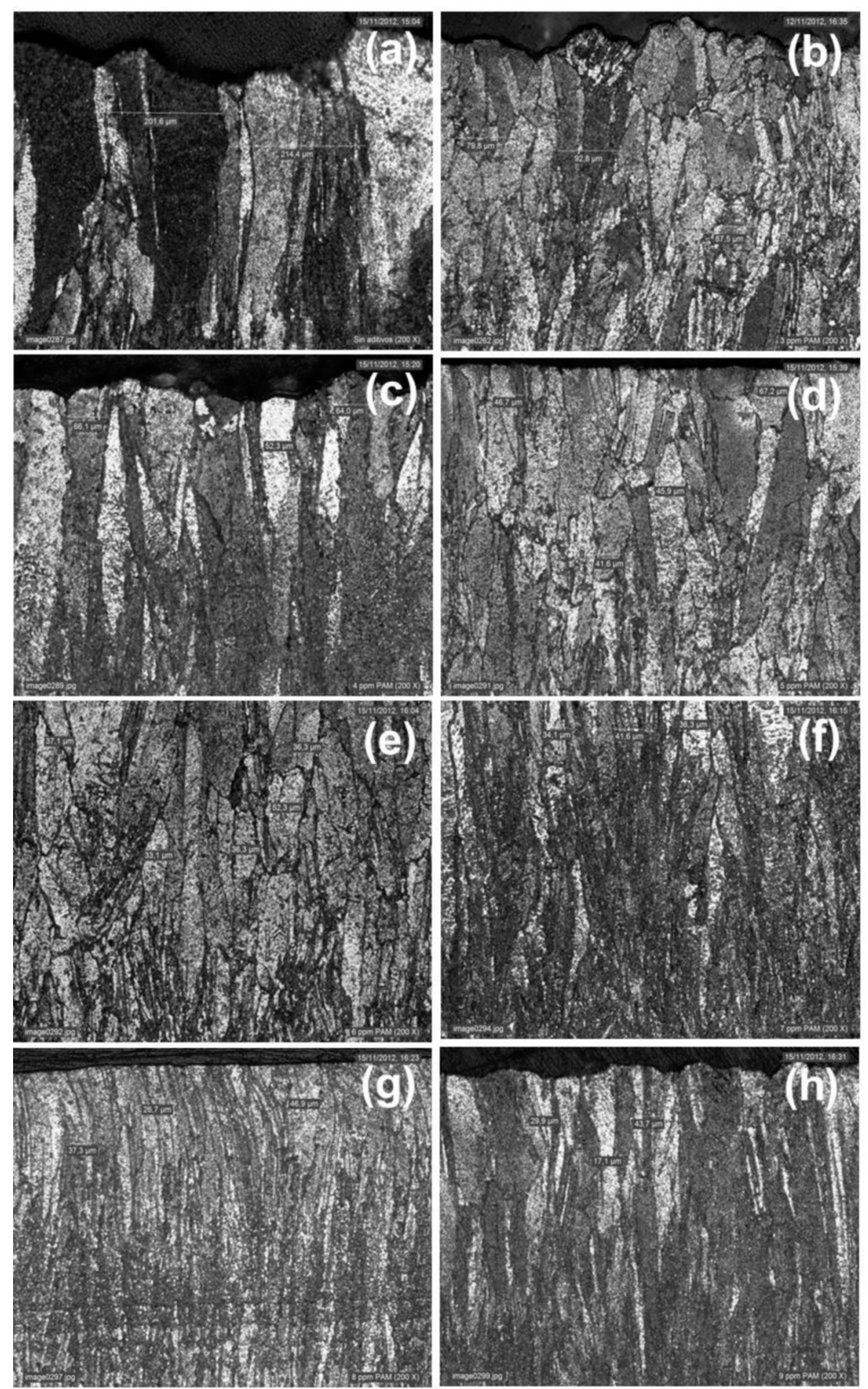

Figura 4. Perfil de crecimiento del depósito de cobre catódico obtenido por microscopía metalográfica con diferentes concentraciones de PAM: (a) sin aditivo; (b) $3 \mathrm{mgl}^{-1}$; (c) $4 \mathrm{mgl}^{-1}$; (d) $5 \mathrm{mgl}^{-1}$; (e) $6 \mathrm{mgl}^{-1}$; (f) $7 \mathrm{mgl}^{-1}$; (g) $8 \mathrm{mgl}^{-1}$; (h) $9 \mathrm{mgl}^{-1} \mathrm{y}$ (i) $10 \mathrm{mgl}^{-1}$.

Figure 4. Growth profile of the copper cathodic deposit obtained by metalographic microscopy with different concentrations of PAM: (a) without additive; (b) $3 \mathrm{mgl}^{-1}$; (c) $4 \mathrm{mgl}^{-1}$; (d) $5 \mathrm{mgl}^{-1}$; (e) $6 \mathrm{mgl}^{-1}$; (f) $7 \mathrm{mgl}^{-1}$; (g) $8 \mathrm{mgl}^{-1}$; (h) $9 \mathrm{mgl}^{-1}$ and (i) $10 \mathrm{mgl}^{-1}$. 


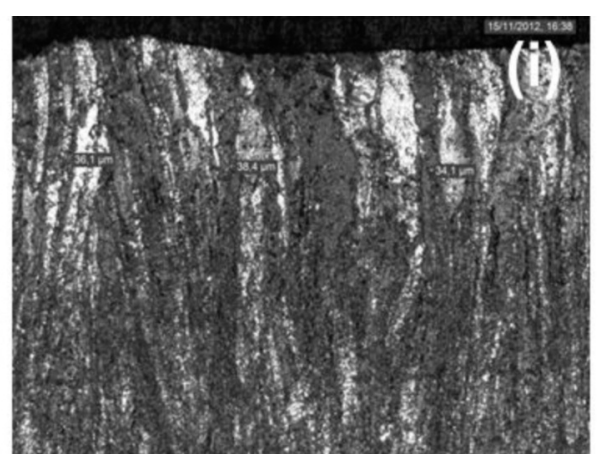

Figura 4 (continuación). (i) $10 \mathrm{mgl}^{-1}$.

Figure 4 (continuation). (i) $10 \mathrm{mgl}^{-1}$.

embargo, no se alcanza la afinación del grano cristalino lograda con $8 \mathrm{mgl}^{-1}$ de PAM (Fig. 4 (g)), pero son satisfactorias, si se las compara con la estructura obtenida con $4 \mathrm{mgl}^{-1}$ de galactasol (Fig. 5).

Las medidas de la rugosidad de la superficie de las láminas catódicas de cobre (Fig. 6), son coherentes con los resultados obtenidos anteriormente. Los valores promedios de $\mathrm{Ra}$ y $\mathrm{R} z$, correspondiente a los promedios de las zonas del lado centro, izquierdo y derecho de cada cara, indican que los menores valores en el depósito de cobre se obtienen con $8 \mathrm{mgl}^{-1}$ de PAM, siendo significativamente menores a los obtenidos con $4 \mathrm{mgl}^{-1}$ de galactasol.

Los valores promedios mínimos de $\mathrm{Ra}$ y $\mathrm{Rz}$ (6 y $40 \mu \mathrm{m}$, respectivamente), se relacionan con concentraciones de 8 a $10 \mathrm{mgl}^{-1}$ de PAM y de $4 \mathrm{mgl}^{-1}$ de

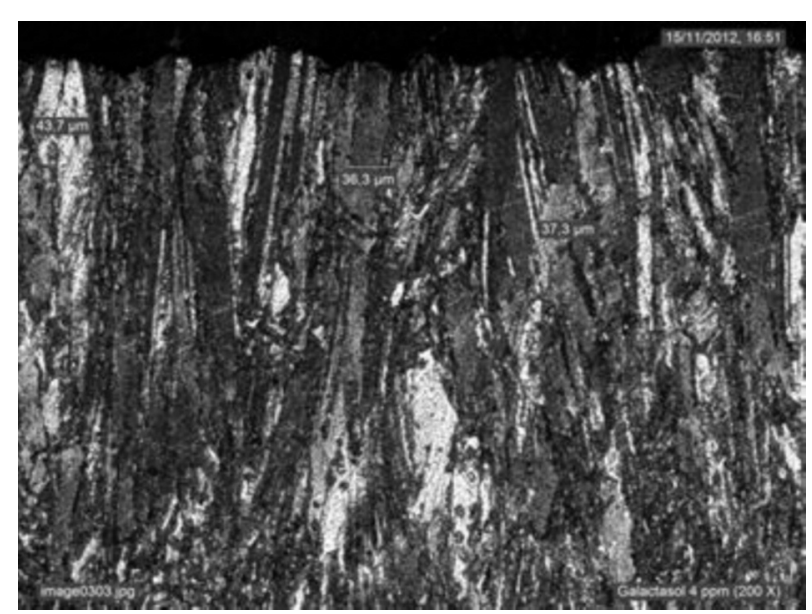

Figura 5. Perfil de crecimiento del depósito de cobre catódico obtenido por microscopía metalográfica con $4 \mathrm{mgl}^{-1}$ de galactasol.

Figure 5. Growth profile of the copper cathodic deposit obtained by metalographic microscopy with $4 \mathrm{mgl}^{-1}$ of galactasol. guar (Galactasol@), mientras que los valores de Ra y $\mathrm{Rz}$ sin aditivos, son de 15 y $100 \mu \mathrm{m}$, respectivamente. En consecuencia, los valores de $\mathrm{Ra}$ y $\mathrm{Rz}$ disminuyen con el incremento de la concentración de aditivo afinador de grano, modificando significativamente las características físicas del depósito superficial de cobre.

\subsection{Curvas de polarización}

La figura 7 muestra las curvas de polarización para la reacción de reducción del ión cúprico, obtenidas con aditivo PAM en el rango de concentración evaluado, comparándolas con las curvas obtenidas sin aditivo y con $4 \mathrm{mgl}^{-1}$ de galactasol. Se observa que la polarización aumenta con el incremento de la concentración de PAM. Al comparar las curvas obtenidas a diferentes concentraciones de PAM, se observa un incremento del sobrepotencial conforme aumenta la concentración del aditivo, pero ninguna sobrepasa la curva de polarización asociada al galactasol.

\section{CONCLUSIONES}

- La adición de pequeñas cantidades de PAM (del orden de $3 \mathrm{mgl}^{-1}$ ) produce una notable disminución del tamaño de grano cristalino del depósito catódico de cobre. El efecto más significativo se alcanza en el rango de 7 a $10 \mathrm{mgl}^{-1}$ de PAM, obteniéndose una condición óptima a $8 \mathrm{mg} \mathrm{l}^{-1}$ con una estructura de grano fino, uniforme y compacto, con una clara orientación en el campo eléctrico, característico del depósito tipo FT en la clasificación de Fischer, de calidad física superior en comparación con el perfil de crecimiento del depósito catódico obtenido con la adición de guar (Galactasol®). 


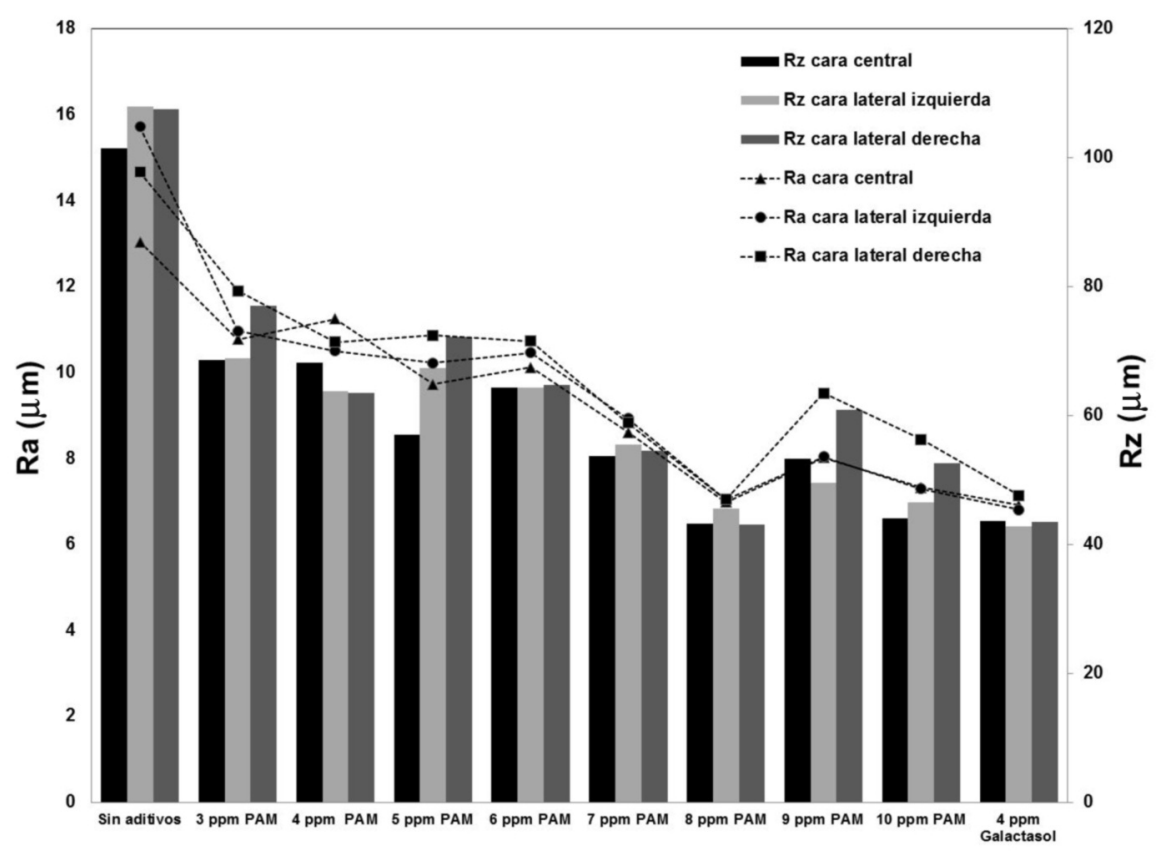

Figura 6. Perfil de rugosidades para Ra y Rz en función de la concentración de aditivo (PAM y galactasol).

Figure 6. Profile of roughness for $R a$ and $R z$ in function of the concentration of additive (PAM and galactasol).

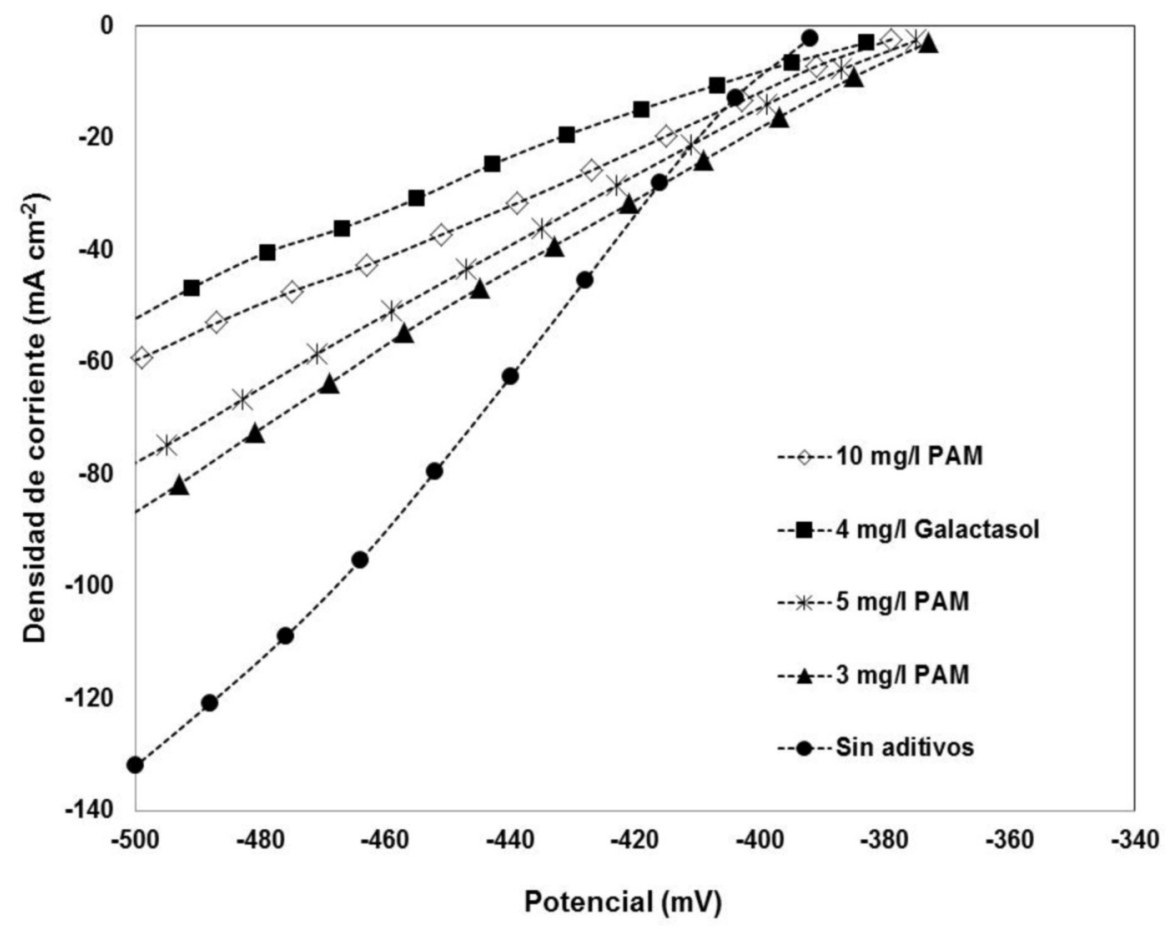

Figura 7. Curvas de polarización sin aditivos, con guar y con diferentes concentraciones de PAM.

Figure 7. Polarization curves without additives, with guar gum and with different concentrations of PAM. 
- El análisis MEB, fue coincidente con los resultados del análisis metalográfico, evidenciando que en el rango de 8 y $10 \mathrm{mgl}^{-1}$ de PAM, se obtienen superficies catódicas caracterizadas por una estructura de grano más fino y compacto, similares a las logradas en electrolitos con $4 \mathrm{mgl}^{-1}$ de guar (Galactasol@), alcanzando un tamaño de grano de diámetro promedio cercano a los $60 \mu \mathrm{m}$.

- Los valores de Ra y $\mathrm{Rz}$ disminuyen con el incremento de la concentración de aditivo afinador de grano, modificando significativamente las características físicas del depósito superficial de cobre (grano fino y superficie uniforme), logrando la compactación y fineza del grano requerida.

- La polarización de la semi-reacción de reducción catódica con $4 \mathrm{mgl}^{-1}$ de guar (Galactasol®) es mayor que la obtenida con $10 \mathrm{mgl}^{-1}$ de PAM.

\section{Agradecimientos}

Los autores agradecen a la Dirección de Investigación de la Universidad Arturo Prat (Chile), por el financiamiento de este estudio.

\section{REFERENCIAS}

[1] T.O' Keefe, Application of Polarisation Measurements in the Control of Metal Deposition, I.H. Warren (Ed.), Elsevier Science Publishers, Amesterdam, Holanda, 1984, pp. $15-31$.

[2] S. Afifi, A. Elsayed y A. Elsherief, JOM 39 (1987) 38-41.
[3] Z. Mubarok, I. Filzwieser y P. Pashen, Proceeding/EMC 2005, Dresden, Alemania, 2005, pp. 109-122.

[4] R. Winand, Application of Polarisation Measurements in the Control of Metal Deposition, I. H. Warren (Ed.), Elsevier, Amsterdam, Holanda, 1984, pp. 47-83.

[5] P. Stanke, Procs. COPPER'99, Vol. III, Electrorefining \& Electrowinning, Minerals, Metals, and Materials Society (Eds.), Phoenix, Arizona, EE.UU., 1999, pp. 643-651.

[6] M. Minakshi y M. Nicol, Proc. Hydrocopper 2009, E. Domic y J. Casas (Eds.), Antofagasta, Chile, 2009, pp. 382-391.

[7] L. Muresan, S. Varvara, G. Maurin y S. Dorneanu, Hydrometallurgy 2-3 (2000) 161-169.

[8] R. Yu, Q. Liu, G. Qiu, Z. Fang, J. Tan y P. Yang, T. Nonferr. Metal. Soc. 5 (2008) 1.2801.284 .

[9] G. Hope y G. Brown, Proc. 189th ECS Meeting, Symp., R. Ruoff y K. Kadish (Eds), Los Angeles, EE.UU., 1996, p. 1.097.

[10] S. Sandoval, C. Morales y C. Bernu, Proc. SME Annual Meeting and Exhibit 2010, Arizona, EE.UU., 2010, p. 540.

[11] Great Process Ltda. Regulador de grano DXG-F7, Ficha Técnica, www.gprocess.cl, Antofagasta, Chile.

[12] J. Vereecken y R. Winand, Surf. Technol. 4 (1976) 227-235.

[13] C. Fabian, M. Ridd y M. Sheehan, Hydrometallurgy 1-2 (2007) 44-55.

[14] C. Fabian, M. Ridd y M. Sheehan, Hydrometallurgy 3-4 (2006) 256-263.

[15] H. Fischer, Plat. Surf. Finish. 56 (1969) 1.2291.233 . 